\title{
GEOMETRIC BOGOMOLOV CONJECTURE FOR NOWHERE DEGENERATE ABELIAN VARIETIES OF DIMENSION 5 WITH TRIVIAL TRACE
}

\author{
KAZUHIKO YAMAKI
}

\begin{abstract}
We prove that the geometric Bogomolov conjecture holds for nowhere degenerate abelian varieties of dimension 5 with trivial trace. By this result together with our previous work, we see that the conjecture holds for an abelian variety such that the difference between the dimension of its maximal nowhere degenerate abelian subvariety and that of its trace equals 5 .
\end{abstract}

\section{INTRODUCTION}

1.1. Geometric Bogomolov conjecture. Let $k$ be an algebraically closed field and let $\mathfrak{B}$ be a normal projective variety. Let $K$ be the function field of $\mathfrak{B}$ unless otherwise specified. We fix an algebraic closure $\bar{K}$ of $K$. Once we take an ample line bundle $\mathcal{H}$ on $\mathfrak{B}$, we have the notion of heights on projective varieties over $\bar{K}$. We refer to [4] for details.

Let $A$ be an abelian variety over $\bar{K}$. Let $L$ be an even ample line bundle on $A$, where "even" means that the pull-back of $L$ by the $(-1)$-times endomorphism is isomorphic to $L$. Then we have the canonical height $\widehat{h}_{L}$ on $A$ associated to $L$, which is known to be a semipositive quadratic form on $A(\bar{K})$. Let $X$ be a closed subvariety of $A$. For any $\epsilon>0$, put

$$
X(\epsilon ; L):=\left\{x \in X(\bar{K}) \mid \widehat{h}_{L}(x) \leq \epsilon\right\} .
$$

Then by [6, Lemma 2.1], the property that $X(\epsilon ; L)$ is dense in $X$ for any $\epsilon>0$ does not depend on the choice of the even ample line bundle $L$. We say that $X$ has dense small points if it has that property (cf. [6, Definition 2.2]).

It would be natural to wish to characterize those closed subvarieties which have dense small points. In the arithmetic setting, some characterization has been established. Indeed, when $K$ is a number field, Zhang has proved in [10] that a closed subvariety has dense small points if and only if it is a torsion subvariety, i.e., the translate of an abelian subvariety by a torsion point. In [5], Moriwaki has generalized Zhang's theorem to the case where $K$ is a finitely generated field over $\mathbb{Q}$, with respect arithmetic heights introduced by himself. In the case of function fields with respect to classical heights, however, the characterization problem is still open. Unlike the arithmetic cases, some abelian varieties over function fields actually have closed subvarieties which are not torsion subvarieties but have dense small points. Taking into account that fact, we have proposed in [6] the following conjecture, called the geometric Bogomolov conjecture.

Date: 23/September/2015, 21:00 GMT+9:00. Version 1.2.

2000 Mathematics Subject Classification. Primary 14G40, Secondary 11G50. 
Conjecture 1.1 (Conjecture 2.9 of [6]). Let $A$ be an abelian variety over $\bar{K}$. Let $X$ be a closed subvariety of $A$. Then $X$ has dense small points if and only if $X$ is a special subvariety.

Here, we recall the definition of special subvarieties defined in [6]. To define them, we use the $\bar{K} / k$-trace of $A$. A pair $\left(\widetilde{A^{K} / k}, \operatorname{Tr}_{A}\right)$ consisting of an abelian variety $\widetilde{A^{K} / k}$ over $k$ and a homomorphism $\operatorname{Tr}_{A}: \widetilde{A^{K} / k} \otimes_{k} \bar{K} \rightarrow A$ of abelian varieties over $\bar{K}$ is called a $\bar{K} / k$-trace of $A$ if it has the following property: For any abelian variety $\widetilde{B}$ over $k$ and a homomorphism $\phi: \widetilde{B} \otimes_{k} \bar{K} \rightarrow A$, there exists a unique homomorphism $\operatorname{Tr}(\phi): \widetilde{B} \rightarrow \widetilde{A^{K} / k}$ such that $\phi$ factors as $\phi=\operatorname{Tr}_{A} \circ\left(\operatorname{Tr}(\phi) \otimes_{k} \bar{K}\right)$. It is unique by the universal property, and it is also known to exist. We call $\operatorname{Tr}_{A}$ the $\bar{K} / k$-trace homomorphism of $A$. We refer to [3] for more details. Then, a closed subvariety $X$ of $A$ is said to be special if there exists a closed subvariety $\widetilde{Y}$ of $\widetilde{A}^{\bar{K} / k}$ and a torsion subvariety $T$ of $A$ such that $X=\operatorname{Tr}_{A}\left(\widetilde{Y} \otimes_{k} \bar{K}\right)+T$.

Remark that one easily sees that the "if" part of the geometric Bogomolov conjecture holds (cf. [6, Corollary 2.8]), and the essential part is the "only if" part.

Although Conjecture 1.1 is still open in full generality, there are some partial answers. In [1], Gubler has proved the conjecture holds under the assumption that $A$ is totally degenerate at some place. In [7], we have generalized Gubler's work and have shown that the conjecture for $A$ is reduced to the conjecture for its maximal nowhere degenerate abelian subvariety $\mathfrak{m}$, where "being nowhere degenerate" means "having everywhere good reduction" and "maximal" means "maximal with respect to the inclusion" (cf. [7, Definition 7.10]). It is known that there exists such an $\mathfrak{m}$ uniquely. In [8], we have proved the following theorem, where $\mathfrak{t}$ is the image of the $\bar{K} / k$-trace homomorphism $\operatorname{Tr}_{A}$ of $A$, and it is known to be contained in $\mathfrak{m}$ (cf. [7, Lemma $7.8(2)]$ ).

Theorem 1.2 (cf. Theorem 1.5 of [8]). With the notation above, the following are equivalent to each other:

(a) The geometric Bogomolov conjecture holds for $A$;

(b) The geometric Bogomolov conjecture holds for $\mathfrak{m} / \mathfrak{t}$.

Remark that in a recent paper [9], we have shown that if $X$ is a closed subvariety having dense small points such that $\operatorname{dim}(X)=1$ or $\operatorname{codim}(X, A)=1$, then it is a special subvariety. That result will be used in the arguments in this note.

1.2. Results. In this note, we prove that Conjecture 1.1 holds for nowhere degenerate abelian varieties of dimension 5 with trivial $\bar{K} / k$-trace:

Theorem 1.3. Let $A$ be a nowhere degenerate abelian variety over $\bar{K}$ of dimension 5 with trivial $\bar{K} / k$-trace. Let $X$ be a closed subvariety of $A$. Suppose that $X$ has dense small points. Then $X$ is a torsion subvariety.

Theorem 1.3 contributes the geometric Bogomolov conjecture not only for nowhere degenerate abelian varieties but also for more general abelian varieties. Let $A$ be any abelian variety over $\bar{K}$. Let $\mathfrak{m}$ and $\mathfrak{t}$ be as above. Since $\mathfrak{m} / \mathfrak{t}$ is a nowhere degenerate abelian variety with trivial $\bar{K} / k$-trace (cf. [7, Lemma 7.8 (2)] and [8, Remark 5.4]), the following theorem is deduced from Theorem 1.3 and Theorem [1.2. 
Theorem 1.4. Let $A, \mathfrak{m}$ and $\mathfrak{t}$ be as above. Assume that $\operatorname{dim}(\mathfrak{m} / \mathfrak{t})=5$. Then the geometric Bogomolov conjecture holds for $A$.

In [9], we show that the geometric Bogomolov conjecture holds for $A$ with $\operatorname{dim}(\mathfrak{m} / \mathfrak{t}) \leq 3$. It follows by Theorem 1.4 that the conjecture holds for $A \operatorname{such}$ that $\operatorname{dim}(\mathfrak{m} / \mathfrak{t}) \leq 5$ and $\operatorname{dim}(\mathfrak{m} / \mathfrak{t}) \neq 4$. At this moment, we do not have an idea for $\operatorname{dim}(\mathfrak{m} / \mathfrak{t})=4$.

The proof of Theorem 1.3 relies heavily on the results in [9], that is, the non-density of small points on closed subvarieties of dimension 1 or of codimension 1 . The key tools to deduce the theorem from this non-density result are the morphisms $X^{m} \rightarrow A^{m-1}$ given by $\left(x_{1}, \ldots, x_{m}\right) \mapsto\left(x_{1}-x_{2}, x_{2}-x_{3}, \ldots, x_{m-1}-x_{m}\right)$ for $m=2,3$.

Acknowledgments. This work was initiated during my visit to the university of Regensburg in March-April 2015, which was supported by the SFB Higher Invariants. I thank Professor Walter Gubler for inviting me and for his hospitality. I thank him also for his comments. This work was partly supported by KAKENHI 26800012.

\section{PROOF}

In this article, a variety means a non-empty geometrically integral scheme which is separated and of finite type over a field.

2.1. Difference morphism. In this subsection, let $A$ be an abelian variety over an algebraically closed field $\mathfrak{K}$. Let $X$ be a closed subvariety of $A$. For any $x \in X(\mathfrak{K})$, let $G(x)$ be the closed subset of $A$ defined by

$$
G(x):=\{a \in A \mid a+x \in X\} .
$$

For $x_{1}, \ldots, x_{m} \in X(\mathfrak{K})$, we set $G\left(x_{1}, \ldots, x_{m}\right):=G\left(x_{1}\right) \cap \cdots \cap G\left(x_{m}\right)$. Let $G_{X}$ be the stabilizer of $X$. Then $G_{X}=\bigcap_{x \in X(\mathfrak{K})} G(x)$.

For any integer $m$ with $m \geq 2$, let $\alpha_{X^{m}}: X^{m} \rightarrow A^{m-1}$ be the morphism given by

$$
\alpha_{X^{m}}:\left(x_{1}, \ldots, x_{m}\right) \mapsto\left(x_{1}-x_{2}, x_{2}-x_{3}, \ldots, x_{m-1}-x_{m}\right) .
$$

These morphisms $\alpha_{X^{m}}$ for $m \geq 2$, called difference morphisms, will play important roles.

The following assertion is stated in the proof of [10, Lemma 3.1]. It can be also found at [2, Claim 6.2.2.1] with a proof.

Lemma 2.1. For any $\left(x_{1}, \ldots, x_{m}\right) \in X^{m}(\mathfrak{K})$, we have

$$
\left(\alpha_{X^{m}}\right)^{-1}\left(\alpha_{X^{m}}\left(x_{1}, \ldots, x_{m}\right)\right)=\left\{\left(x_{1}+a, \ldots, x_{m}+a\right) \mid a \in G\left(x_{1}, \ldots, x_{m}\right)\right\} .
$$

In particular, $\operatorname{dim}\left(\left(\alpha_{X^{m}}\right)^{-1}\left(\alpha_{X^{m}}\left(x_{1}, \ldots, x_{m}\right)\right)\right)=\operatorname{dim}\left(G\left(x_{1}, \ldots, x_{m}\right)\right)$.

We set

$$
d_{m}(X):=\min \left\{\operatorname{dim}\left(G\left(x_{1}, \ldots, x_{m}\right)\right) \mid x_{1}, \ldots, x_{m} \in X(\mathfrak{K})\right\},
$$

where "dim" means the maximum of the dimensions of the irreducible components. Remark that $d_{m}(X) \leq \operatorname{dim}\left(G\left(x_{1}, \ldots, x_{m}\right)\right)$ for any $\left(x_{1}, \ldots, x_{m}\right) \in X^{m}(\mathfrak{K})$. Remark also that $d_{m}(X)=\operatorname{dim}\left(G\left(x_{1}, \ldots, x_{m}\right)\right)$ for general $\left(x_{1}, \ldots, x_{m}\right) \in X^{m}(\mathfrak{K})$ by Lemma 2.1 .

The following lemma is the key in our proof of the theorem.

Lemma 2.2. Suppose that $d_{m}(X)>\operatorname{dim}\left(G_{X}\right)$. Then $d_{m+1}(X)<d_{m}(X)$. 
Proof. Fix an $\left(x_{1}, \ldots, x_{m}\right) \in X(\mathfrak{K})$ with $\operatorname{dim}\left(G\left(x_{1}, \ldots, x_{m}\right)\right)=d_{m}(X)$. Let $Z_{1}, \ldots, Z_{p}$ be the irreducible components of $G\left(x_{1}, \ldots, x_{m}\right)$ of dimension $d_{m}(X)$. For any $j=1, \ldots, p$, let $\psi_{j}: Z_{j} \times X \rightarrow A$ be the restriction of the addition $A^{2} \rightarrow A$ and set $W_{j}:=\psi_{j}\left(Z_{j} \times X\right)$. Since $\operatorname{dim}\left(Z_{j}\right)=d_{m}(X)>\operatorname{dim}\left(G_{X}\right)$, we have $Z_{j} \nsubseteq G_{X}$. It follows that $W_{j} \nsubseteq X$, and hence $\psi_{j}^{-1}(X) \subsetneq Z_{j} \times X$. Therefore, $\operatorname{pr}_{2}\left(\left(Z_{j} \times X\right) \backslash \psi_{j}^{-1}(X)\right)$, where $\operatorname{pr}_{2}: A \times X \rightarrow X$ is the second projection, is a dense open subset of $X$. Thus $U:=\bigcap_{j=1}^{p} \operatorname{pr}_{X}\left(\left(Z_{j} \times X\right) \backslash \psi_{j}^{-1}(X)\right)$ is a dense open subset of $X$.

Take any $x_{m+1} \in U(\mathfrak{K})$. Then for any $j=1, \ldots, p$, there exists $a_{j} \in Z_{j}(\mathfrak{K})$ such that $\left(a_{j}, x_{m+1}\right) \notin \psi_{j}^{-1}(X)$, which means $a_{j} \notin G\left(x_{m+1}\right)$. Thus for any $j=1, \ldots, p$, we have $G\left(x_{m+1}\right) \nsupseteq Z_{j}$. It follows that $G\left(x_{1}, \ldots, x_{m}, x_{m+1}\right)=G\left(x_{1}, \ldots, x_{m}\right) \cap G\left(x_{m+1}\right)$ does not contain any irreducible component of $G\left(x_{1}, \ldots, x_{m}\right)$ of dimension $d_{m}(X)$, which implies that $\operatorname{dim}\left(G\left(x_{1}, \ldots, x_{m}, x_{m+1}\right)\right)<d_{m}(X)$. This shows $d_{m+1}(X)<d_{m}(X)$.

The following lemma characterizes translates of abelian subvarieties in terms of the difference morphism.

Lemma 2.3. Suppose that $\operatorname{dim}\left(\alpha_{X^{2}}\left(X^{2}\right)\right)=\operatorname{dim}(X)$. Then $X$ is a translate of an abelian subvariety.

Proof. Fix a closed point $x_{0} \in X$ and set $Z=X-x_{0}$. It then suffices to show that $Z-Z \subset Z$. First, we note that $Z-Z=X-X=\alpha_{X^{2}}\left(X^{2}\right)$ and that $Z=\alpha_{X^{2}}\left(X \times\left\{x_{0}\right\}\right) \subset$ $\alpha_{X^{2}}\left(X^{2}\right)$. Since $\operatorname{dim}\left(\alpha\left(X^{2}\right)\right)=\operatorname{dim}(X)=\operatorname{dim}(Z)$ and $\alpha\left(X^{2}\right)$ is irreducible, it follows that $Z=\alpha\left(X^{2}\right)$, Thus $Z-Z=\alpha_{X^{2}}\left(X^{2}\right)=Z$ as required.

2.2. Proof of the main theorem. For the proof of Theorem 1.3, we show three lemmas. First two ones would be more or less known facts.

Lemma 2.4. Let $A$ be an abelian variety over $\bar{K}$ and let $X$ be a closed subvariety of $A$. Suppose that $X$ is a translate of an abelian subvariety and has dense small points. Then it is a torsion subvariety.

Proof. We take an abelian subvariety $G$ of $A$ and an $a_{0} \in A(\bar{K})$ such that $X=G+a_{0}$. Let $\phi: A \rightarrow A / G$ be the quotient homomorphism. Then $\phi(X)=\left\{\phi\left(a_{0}\right)\right\}$. By $[\underline{6}$, Lemma 2.1], this has dense small points, which means that $\phi\left(a_{0}\right)$ is a torsion point. Therefore, there exists a torsion point $\tau \in A(\bar{K})$ such that $\phi(\tau)=\phi\left(a_{0}\right)$ (cf. the proof of $[6$, Lemma 2.10]). Thus $X=G+\tau$, which completes the proof of the lemma.

Lemma 2.5. Let $A$ be a nowhere degenerate abelian variety over $\bar{K}$ with trivial $\bar{K} / k$-trace. Then $A$ does not have an abelian subvariety of dimension 1.

Proof. To show the lemma by contradiction, suppose that there exists an abelian subvariety $E$ of $A$ with $\operatorname{dim}(E)=1$. By [9, Lemma 5.5], $E$ is nowhere degenerate and has trivial $\bar{K} / k$-trace. By [8, Proposition 2.4], there exist a finite extension $K^{\prime}$ of $K$ in $\bar{K}$, an open subset $\mathfrak{U}$ of $\mathfrak{B}^{\prime}$ with codim $\left(\mathfrak{B}^{\prime} \backslash \mathfrak{U}, \mathfrak{B}^{\prime}\right) \geq 2$ where $\mathfrak{B}^{\prime}$ is the normalization of $\mathfrak{B}$ in $K^{\prime}$, and an abelian scheme $f: \mathscr{E} \rightarrow \mathfrak{U}$ such that $\mathscr{E} \times_{\mathfrak{U}} \operatorname{Spec}(\bar{K}) \cong E$. Since the moduli space of elliptic curves is the affine line, this $\mathscr{E} \rightarrow \mathfrak{U}$ gives rise to a morphism from $\mathfrak{U}$ to the affine line. Since $E$ has trivial $\bar{K} / k$-trace, this morphism is non-constant. Thus we obtain a non-constant regular function $f$ on $\mathfrak{U}$. Since $\mathfrak{B}^{\prime}$ is normal and $\operatorname{codim}\left(\mathfrak{B}^{\prime} \backslash \mathfrak{U}, \mathfrak{B}^{\prime}\right) \geq 2, f$ extends to a 
regular function on $\mathfrak{B}^{\prime}$. However, that is impossible since $\mathfrak{B}^{\prime}$ is projective. Thus the lemma holds.

The other lemma is a restatement of the non-density results in [9] which is modified for our setting.

Lemma 2.6. Let $A$ be a nowhere degenerate abelian variety over $\bar{K}$ with trivial $\bar{K} / k$-trace and let $X$ be a closed subvariety of $A$. Suppose that $X$ has dense small points. Then $\operatorname{dim}(X) \neq 1$ and $\operatorname{codim}(X, A) \neq 1$.

Proof. By [9, Proposition 5.6], $X$ is not a divisor, and thus codim $(X) \neq 1$. We show that $\operatorname{dim}(X) \neq 1$ by contradiction. Suppose that $\operatorname{dim}(X)=1$. By [9, Theorem 5.10], $X$ is a special subvariety of $A$. Since $A$ has trivial $\bar{K} / k$-trace, that implies that $X$ is a torsion subvariety of dimension 1 , and thus there exists an abelian subvariety $E$ of $A$ with $\operatorname{dim}(E)=1$. However, that contradicts Lemma 2.5.

We now restate the main theorem and prove it.

Theorem 2.7 (Theorem 1.3). Let $A$ be a nowhere degenerate abelian variety of dimension 5 with trivial $\bar{K} / k$-trace. Let $X$ be a closed subvariety of $A$. Suppose that $X$ has dense small points. Then $X$ is a torsion subvariety.

Proof. The case of $\operatorname{dim}(X)=5$ is trivial, and the case of $\operatorname{dim}(X)=0$ is classically known (cf. [7, Remark 7.4]). Further by Lemma 2.6, we have $\operatorname{dim}(X) \neq 1$ and $\operatorname{dim}(X) \neq 4$. Thus we may assume that $\operatorname{dim}(X)=2,3$.

To ease notation, we write $\alpha_{m}$ for $\alpha_{X^{m}}$. We set $Y_{2}:=\alpha_{2}\left(X^{2}\right) \subset A$. Since $X^{2}$ has dense small points, so does $Y_{2}$. By Lemma 2.6, we have $\operatorname{dim}\left(Y_{2}\right) \neq 4$.

First, we consider the case of $\operatorname{dim}(X)=3$. We prove $\operatorname{dim}\left(Y_{2}\right) \neq 5$ then. To argue by contradiction, suppose that $\operatorname{dim}\left(Y_{2}\right)=5$. Then $\alpha_{2}: X^{2} \rightarrow A$ is surjective. Since $\operatorname{dim}\left(X^{2}\right)-\operatorname{dim}(A)=1$, we have $d_{2}(X)=1$ (cf. Lemma 2.1). Remark in particular that $\operatorname{dim}\left(G_{X}\right) \leq 1$. By Lemma 2.5, we find $\operatorname{dim}\left(G_{X}\right)=0$. By Lemma 2.2, it follows that $d_{3}(X)=0$. This means that $\alpha_{3}: X^{3} \rightarrow A^{2}$ is generically finite, and hence $\operatorname{dim}\left(\alpha_{3}\left(X^{3}\right)\right)=$ $3 \operatorname{dim}(X)=9$. Thus $\alpha_{3}\left(X^{3}\right)$ is an effective divisor on $A$. On the other hand, since $X$ has dense small points, so does $X^{3}$, and hence $\alpha_{3}\left(X^{3}\right)$ has dense small points. However, since $A^{3}$ is nowhere degenerate and has trivial $\bar{K} / k$-trace (cf. [9, Lemma 5.5]), that contradicts Lemma 2.6. Thus we obtain $\operatorname{dim}\left(Y_{2}\right) \neq 5$.

Since $\operatorname{dim}\left(Y_{2}\right) \geq \operatorname{dim}(X)=3$, it follows that $\operatorname{dim}\left(Y_{2}\right)=3$. Then Lemma 2.3 shows that $X$ is a translate of an abelian subvariety. Since $X$ has dense small points, it follows by Lemma 2.4 that $X$ is a torsion subvariety. Thus we obtain the theorem in the case of $\operatorname{dim}(X)=3$.

Next, we consider the case of $\operatorname{dim}(X)=2$. Since $\operatorname{dim}\left(Y_{2}\right) \neq 4$, we then have $2 \leq \operatorname{dim}\left(Y_{2}\right) \leq$ 3. In fact, we prove $\operatorname{dim}\left(Y_{2}\right)=2$. To argue by contradiction, suppose that $\operatorname{dim}\left(Y_{2}\right)=3$. Since $Y_{2}$ is a closed subvariety of $A$ having dense small points with $\operatorname{dim}\left(Y_{2}\right)=3$, it follows from what we have shown above that $Y_{2}$ is a torsion subvariety. Since $0 \in Y_{2}, Y_{2}$ is an abelian subvariety. By the Poincaré complete reducibility theorem, therefore, there exists an abelian subvariety $A^{\prime}$ of $A$ of dimension 2 such that $Y_{2}+A^{\prime}=A$. Let $\phi: A \rightarrow A / A^{\prime}$ be the quotient homomorphism. Then $A / A^{\prime}$ is an abelian variety of dimension 3 , and furthermore by [9, Lemma 5.5], it is nowhere degenerate and has trivial $\bar{K} / k$-trace. Since $Y_{2} \cap A^{\prime}$ is finite 
and since some translate of $X$ lies in $Y_{2}$, we see that $\operatorname{dim}(\phi(X))=\operatorname{dim}(X)=2$. Thus $\phi(X)$ is a divisor on $A / A^{\prime}$. On the other hand, since $X$ has dense small points, so does $\phi(X)$. However, that is a contradiction by Lemma 2.6.

Thus we have $\operatorname{dim}\left(Y_{2}\right)=2$. By Lemma 2.3, it follows that $X$ is a translate of an abelian subvariety. Since $X$ has dense small points, Lemma 2.4 concludes that $X$ is a torsion subvariety. Thus we complete the proof.

Finally, as is noted in the introduction, Theorem 1.4 is deduced from Theorem 2.7.

\section{REFERENCES}

[1] W. Gubler, The Bogomolov conjecture for totally degenerate abelian varieties, Invent. Math. 169 (2007), $377-400$.

[2] S. Kawaguchi, A. Moriwaki, K. Yamaki, Introduction to Arakelov geometry, in Algebraic geometry in East Asia (Kyoto, 2001), 1-74, World Sci. Publ.

[3] S. Lang, Abelian varieties, Springer-Verlag (1983).

[4] S. Lang, Fundamentals of Diophantine Geometry, Springer-Verlag (1983).

[5] A. Moriwaki, Arithmetic height functions over finitely generated fields, Invent. Math. 140 (2000), 101142.

[6] K. Yamaki, Geometric Bogomolov conjecture for abelian varieties and some results for those with some degeneration (with an appendix by Walter Gubler: The minimal dimension of a canonical measure), Manuscr. Math. 142 (2013), 273-306.

[7] K. Yamaki, Strict support of canonical measures and applications to the geometric Bogomolov conjecture, to appear in Compositio Mathematica, arXiv:1211.0406v4.

[8] K. Yamaki, Trace of abelian varieties over function fields and the geometric Bogomolov conjecture, to appear in Journal für die reine und angewandte Mathematik, arXiv:1405.0896v4.

[9] K. Yamaki, Non-density of small points on divisors on abelian varieties and the Bogomolov conjecture, preprint, arXiv:1505.03665v2.

[10] S. Zhang, Equidistribution of small points on abelian varieties, Ann. of Math. (2) 147 (1998), no. 1, 159-165.

Institute for Liberal Arts And Sciences, Kyoto University, Kyoto, 606-8501, Japan

E-mail address: yamaki.kazuhiko.6r@kyoto-u.ac.jp 